ARTICLE

httpst//doi.org/10.1038/s414.67-020-15036-y

\title{
Tailoring the separation properties of flexible metal-organic frameworks using mechanical pressure
}

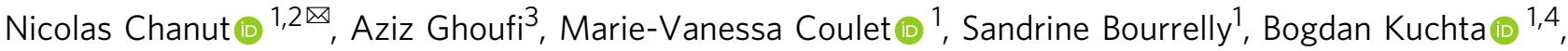 \\ Guillaume Maurin $^{5} \&$ Philip L. Llewellyn (1) ${ }^{1}$
}

Metal-organic frameworks are widely considered for the separation of chemical mixtures due to their adjustable physical and chemical properties. However, while much effort is currently devoted to developing new adsorbents for a given separation, an ideal scenario would involve a single adsorbent for multiple separations. Porous materials exhibiting framework flexibility offer unique opportunities to tune these properties since the pore size and shape can be controlled by the application of external stimuli. Here, we establish a proof-of-concept for the molecular sieving separation of species with similar sizes $\left(\mathrm{CO}_{2} / \mathrm{N}_{2}\right.$ and $\left.\mathrm{CO}_{2} / \mathrm{CH}_{4}\right)$, via precise mechanical control of the pore size aperture in a flexible metal-organic framework. Besides its infinite selectivity for the considered gas mixtures, this material shows excellent regeneration capability when releasing the external mechanical constraint. This strategy, combining an external stimulus applied to a structurally compliant adsorbent, offers a promising avenue for addressing some of the most challenging gas separations.

\footnotetext{
${ }^{1}$ Aix-Marseille University, CNRS Laboratoire MADIREL (UMR7246), Marseille, France. ${ }^{2}$ Massachusetts Institute of Technology, MultiScale Materials Science for Energy \& Environment <MSE 2>, MIT-CNRS-AMU joint laboratory/MIT Energy Initiative, Cambridge 02139 MA, USA. ${ }^{3}$ Institut de Physique de Rennes, IPR UMR 6251 CNRS, Rennes, France. ${ }^{4}$ Department of Chemistry, Wroclaw University of Science and Technology, Wroclaw, Poland. ${ }^{5}$ Institut

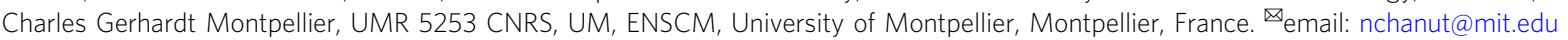


W ith an estimation that $10-15 \%$ of the world's energy resources are used for separations of chemical mixtures $^{1}$, it is clear that any improvement of these processes can have a significant impact from an economic and environmental standpoint. Gas separation and purification steps are particularly energy demanding, especially when the species to be separated exhibit similar physical and chemical/electronic properties. With a potential reduction in energy consumption estimated at $80 \%$, adsorption-based technologies using porous solids or membranes are considered as an attractive alternative to costly distillation and absorption processes ${ }^{2}$.

The viability of adsorption-based separation processes relies on the development of adsorbents with specific physical and chemical properties, controlling the interactions with the molecules in the mixture to be separated. Depending on the process envisaged, adsorption-based separations can be ruled by various mechanisms: (i) enthalpic, where the separation is driven by the difference in affinities of the components in a mixture towards a given adsorbent (ii) kinetic, where the separation depends on the difference in diffusion rates of the various species through the porous material (iii) entropic, where the shape of the pores of the adsorbent proffers an optimal packing of a given molecule and (iv) molecular sieving, where the adsorbent has pores of specific dimensions that allow small molecules to enter whilst excluding larger ones ${ }^{3}$. While for each separation mechanism, specific physical and chemical properties of the adsorbent are required, there is a common quest for an optimal balance between the highest selectivity towards a given species combined with the lowest possible energy required for regeneration, i.e., desorption of confined species from the pores for further re-utilization of the adsorbent. However, the selectivity and regeneration performances are often antagonistic, and this makes the realization of such an optimal scenario quite complicated. From the aforementioned separation mechanisms, molecular sieving can be considered as the ultimate goal, since it allows the complete recovery of one component from a mixture leading to possible ideal selectivity. However, this type of separation is highly challenging to achieve for molecules of similar sizes ${ }^{3}$. One strategy that is currently adopted involves the development of ultramicroporous adsorbents with precisely tailored pore dimensions. Such a family of materials has successfully led to molecular sieving for complex separations of molecules with similar sizes, such as carbon dioxide/nitrogen ${ }^{4}$, propane/propylene ${ }^{5}$, and butane/isobutane $e^{6}$. However, each of these separations is material dependent and requires specific pore dimensions with the design of a novel porous material for each mixture of interest. Furthermore, the high degree of confinement in ultra-microporous adsorbents leads to strong adsorbate/adsorbent interactions. This has two consequences: (i) a significant amount of energy is required to induce gas desorption and (ii) a slow diffusion of species may limit their evacuation, both these effects leading to a costly regeneration of the adsorbent.

Here, we present a strategy where the pore size aperture of a flexible metal-organic framework (MOF) can be finely tuned via the application of external mechanical pressure. Specifically, the possibility to control in situ the guest-assisted breathing behavior of the MIL-53 framework during both the adsorption and desorption steps is presented.

Guest-assisted breathing phenomena in MOFs are well documented $^{7-10}$. These entail through adsorption of a guest molecule, a concerted crystal-to-crystal phase transition between equilibrium structures, e.g., open and contracted phases, generally involving a significant change in the unit cell volume. Initially considered as a fundamental curiosity, this intriguing behavior has attracted much attention lately for both the storage and the separation of molecular species ${ }^{11}$. Further works have shown that such a magnitude of flexibility can equally be induced by other physical stimuli, such as temperature ${ }^{12}$, light ${ }^{13}$, electrical field ${ }^{14}$, and mechanical pressure ${ }^{15}$. In the latter case, some studies have highlighted the possibility of using the mechanically induced flexibility of MOFs to store energy mechanically ${ }^{16-19}$ or manage thermal effects in gas storage application ${ }^{20}$. Nonetheless, to date, no study has explored this concept in a synergistic combination with adsorption for further development in separation-based processes. The concept proposed in this study is to (i) use mechanical pressure to induce the structural transition from an open-pore form of a compliant MOF towards a contracted form, then (ii) proceed to adsorption with optimal conditions of separation, before (iii) switching back to the open pore form by releasing the mechanical pressure for regeneration in a less confined state (Fig. 1a).

\section{Results}

Experimental approach. The porous MOF MIL- $53^{21}$ has been selected as a model candidate to propose a proof-of-concept of this line of thought. This channel-like framework made of chains of metal octahedra sharing $\mu_{2}-\mathrm{OH}$ vertices linked through terephthalate ligands, has been demonstrated to exhibit in its aluminum and chromium forms a guest-assisted reversible phase transition between a large pore (LP) and a narrow pore (NP) phase, accompanied with a remarkable change of the unit cell volume of about $40 \%{ }^{22}$. As illustrated in Fig. $1 \mathrm{~b}$ for $\mathrm{CO}_{2}$ adsorption, this leads to a two-step isotherm. At room temperature and in the absence of guest molecules, the LP phase is the most stable form. A slight increase in pressure induces a narrowing of the pores leading to the NP phase while increasing the $\mathrm{CO}_{2}$ pressure reopens the structure in its LP phase. On desorption, as the $\mathrm{CO}_{2}$ is evacuated from the pores, the reversible structural transition is observed (i.e., LP to NP phase) with hysteresis in the transition pressure.

As a consequence of this phase transition, the pore size aperture varies from $8 \AA$ in the LP phase down to $3.5 \AA$ in the NP phase (Fig. 1c), making this material potentially of interest to molecular sieve $\mathrm{CO}_{2}$ over $\mathrm{N}_{2}$ and $\mathrm{CH}_{4}$ due to the difference in their kinetic diameters $(3.3,3.7 \text {, and } 3.8 \AA \text {, respectively })^{23}$. Furthermore, previous studies have demonstrated that a similar contraction of the structure can be induced by the application of hydrostatic mechanical pressure in the range of $20-100 \mathrm{MPa}^{15,17}$. These structural features, together with the excellent mechanical stability of MIL-53(Al, Cr) up to $500 \mathrm{MPa}^{18}$, make this MOF ideal for the present study. To exploit this concept, we have developed a unique experimental setup allowing the measurement of highpressure gas adsorption isotherms under external, uniaxial mechanical constraint (Supplementary Fig. 1). The use of a hydraulic mechanical press allows for the control of the mechanical pressure, which is maintained constant and continuously applied on the powder bed throughout the adsorption experiment.

Gas adsorption under applied mechanical pressure. Figure 2 reports the adsorption isotherms of $\mathrm{CO}_{2}, \mathrm{~N}_{2}$, and $\mathrm{CH}_{4}$ collected on the MIL-53(Al) constrained to various mechanical pressures at $303 \mathrm{~K}$. The unconstrained MIL-53(Al) presents the abovementioned two-step isotherm for $\mathrm{CO}_{2}$ in agreement with previous studies ${ }^{22}$. Unlike carbon dioxide, nitrogen and methane do not trigger the breathing transition in the temperature and gas pressure conditions investigated here $\left(T=303 \mathrm{~K}\right.$ and $P_{\text {gas }} \leq 15$ bar) and the material remains in its LP phase as previously evidenced by X-ray diffraction (XRD) ${ }^{24}$.

Interestingly, the application of a mechanical pressure of 100 $\mathrm{MPa}$ shows an enhancement of the $\mathrm{CO}_{2}$ uptake by a factor two at 
a
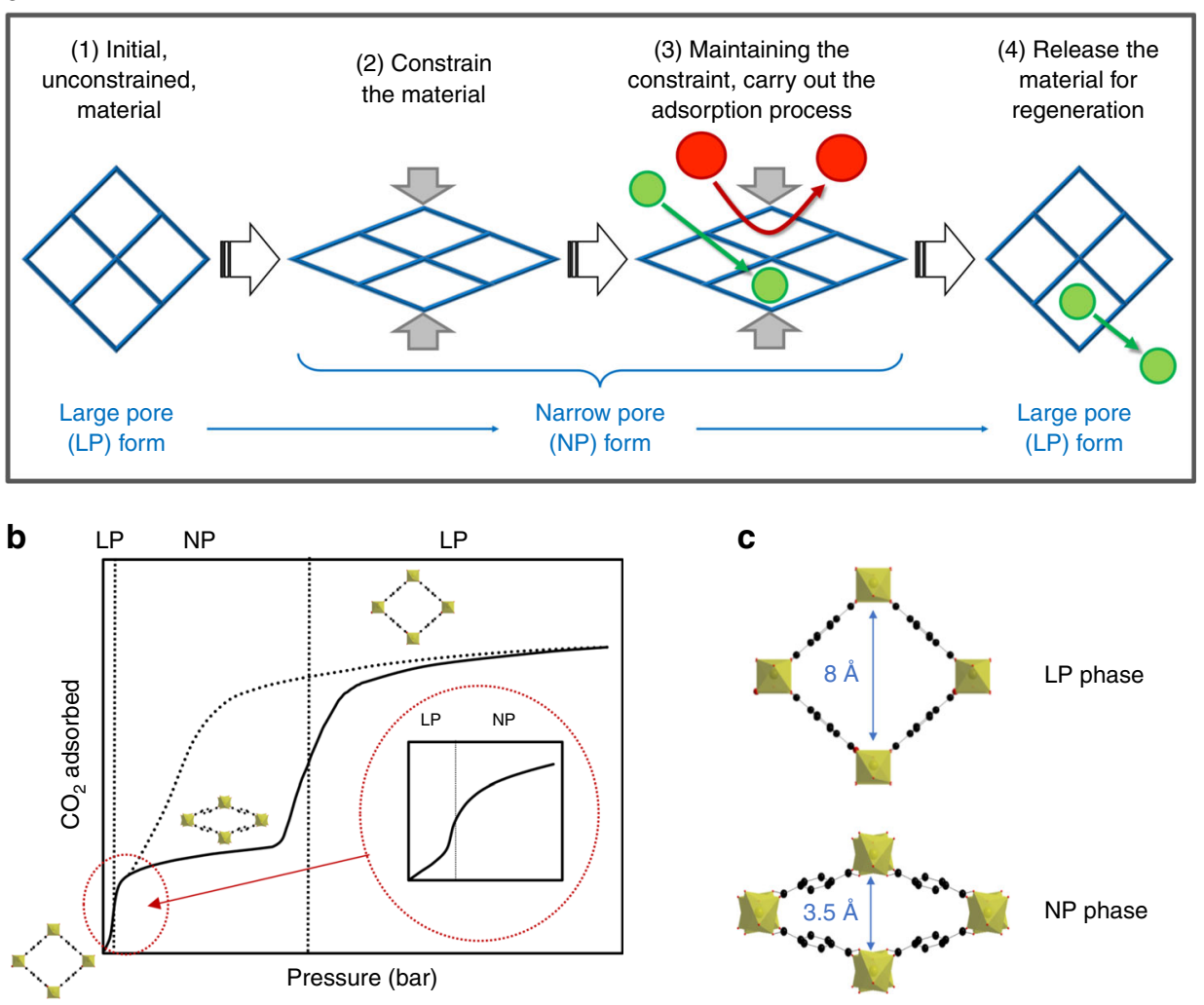

C

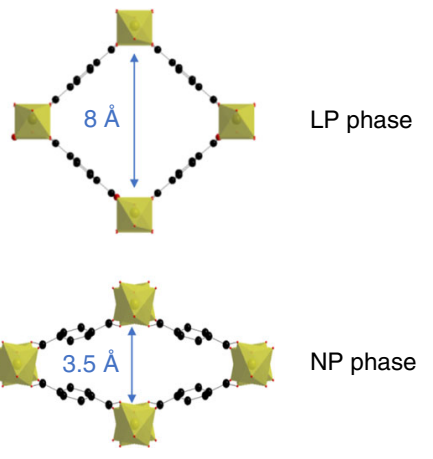

Fig. 1 Flexibility of the MIL-53 framework. a Schematic representation of the concept developed in the present study: control of the breathing behavior of the flexible MIL-53 MOF by application of an external mechanical pressure to provoke a molecular-sieving type separation, followed by regeneration from the unconstrained MOF after release of the mechanical constraint. $\mathbf{b} \mathrm{CO}_{2}$ isotherm (full-line-adsorption, dashes-desorption) of the unconstrained MIL-53 evidencing a two-step process indicative of the framework breathing. Phase transitions between large pore (LP) and narrow pore (NP) phases are indicated by vertical dashed black lines. c Evolution of pore size aperture between the LP and NP phases of MIL-5321.


Fig. 2 Effect of mechanical pressure on $\mathbf{C O}_{\mathbf{2}}, \mathbf{N}_{\mathbf{2}}$, and $\mathbf{C H}_{\mathbf{4}}$ adsorption properties of $\mathbf{M I L}-\mathbf{5 3}(\mathbf{A l})$. a-f Adsorption isotherms of $\mathrm{CO}_{2}$ (a, $\mathbf{d}$ circles), $\mathrm{N}_{2}$ (b, e squares), and $\mathrm{CH}_{4}$ (c, $\mathbf{f}$ triangles) recorded at $303 \mathrm{~K}$ for different applied mechanical pressures. a-c correspond to isotherms recorded up to 1 bar while d-f correspond to isotherms recorded up to 15 bar. Yellow, orange, red, brown, and dark gray filled symbols represent an applied mechanical pressure of 0 , $100,200,300$, and $400 \mathrm{MPa}$, respectively. a Black arrows indicate the evolution of $\mathrm{CO}_{2}$ uptake when gradually increasing the mechanical constraint. 
a

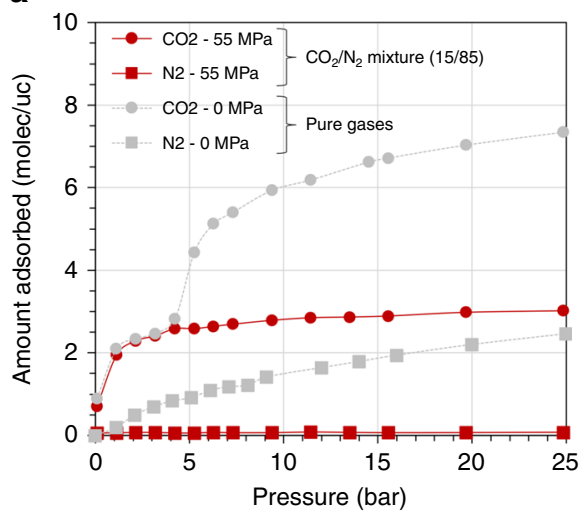

b

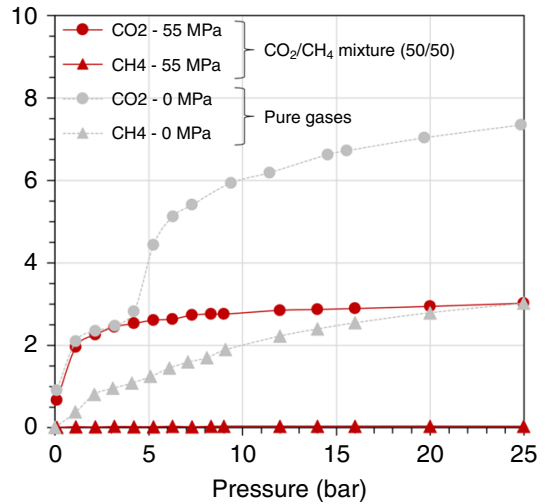

Fig. 3 Hybrid osmotic Monte Carlo (HOMC) simulations of binary mixtures in MIL-53(Cr). a, b Simulated isotherms at $298 \mathrm{~K}$ for a the binary mixture $\mathrm{CO}_{2} / \mathrm{N}_{2}(15 / 85)$ under mechanical constraint (red circles $-\mathrm{CO}_{2}$; red squares $-\mathrm{N}_{2}$ ), and pure $\mathrm{CO}_{2}$ (gray circles) and $\mathrm{N}_{2}$ (gray squares) on the unconstrained MIL-53(Cr) and $\mathbf{b}$ the binary mixture $\mathrm{CO}_{2} / \mathrm{CH}_{4}(50 / 50)$ under mechanical constraint (red circles $-\mathrm{CO}_{2}$; red triangles- $\mathrm{CH}_{4}$ ), and pure $\mathrm{CO}_{2}$ (gray circles) and $\mathrm{CH}_{4}$ (gray triangles) on the unconstrained MIL-53(Cr). Isotherms were obtained by using hybrid osmotic Monte Carlo simulations with an applied isostatic mechanical pressure of 0 and $55 \mathrm{MPa}$ for the unconstrained and mechanically constrained MIL-53(Cr), respectively. The unit of the $y$-axis 'molec/uc' refers to molecules per unit cell.

$P_{\mathrm{CO} 2}$ around 0.2 bar (Fig. 2a). This suggests a transition from the LP to the NP phase, since previous microcalorimetry and molecular modeling studies have shown that the interaction energy of $\mathrm{CO}_{2}$ in the NP phase is significantly higher than in the LP phase, leading to higher uptake at low pressure $22,25-28$. However, it is well documented that for a granular medium under uniaxial constraint, the mechanical pressure is heterogeneously distributed on the different crystallites through the existence of force chains ${ }^{29,30}$. This suggests that not all the crystallites have undergone the LP to NP phase transition and therefore that both phases coexist, which is confirmed by the step in $\mathrm{CO}_{2}$ uptake above $P_{\mathrm{CO} 2}=4-5$ bar (Fig. $2 \mathrm{~d}$ ), the pressure corresponding to the reopening of the structure (NP to LP phase transition).

Interestingly, this step disappears when applying a mechanical pressure of $200 \mathrm{MPa}$. We relate this result to the fact that, under such large mechanical pressure, sintering of the powder occurs, i.e., we observe the irreversible merging of the powder into a pellet. This concerted closure of the crystallites is confirmed by the $\mathrm{N}_{2}$ isotherm collected under $200 \mathrm{MPa}$ (Fig. 3b, e). Significantly, no uptake is observed until $P_{\mathrm{N} 2}$ around $10 \mathrm{bar}$ indicating that all the crystallites are maintained in a form, which does not allow $\mathrm{N}_{2}$ to enter the pores in this gas pressure range. However, beyond 10 bar, the slight increase in $\mathrm{N}_{2}$ uptake is most probably associated with a reopening of certain crystallites. This highlights a competition between the external mechanical pressure that tends to close the pores and the internal pressure due to gas adsorption that would tend to open them. It is thus clear that an equilibrium state exists in the bulk powder that results from a subtle balance between these two effects. It is important to note the excellent reproducibility of the adsorption isotherms after loading/unloading cycles (Supplementary Fig. 2), together with the excellent stability of the structure as verified by the XRD data collected before and after 300 cycles (Supplementary Fig. 3).

Similar behavior to nitrogen is observed for methane adsorption under mechanical pressure. Indeed, the uptake is negligible until (i) $P_{\mathrm{CH} 4}$ around 0.2 bar for a mechanical pressure of $200 \mathrm{MPa}$, (ii) $P_{\mathrm{CH} 4}$ around 2 bar for a mechanical pressure of $300 \mathrm{MPa}$ and (iii) $P_{\mathrm{CH} 4}$ around 5 bar for a mechanical pressure of $400 \mathrm{MPa}$ (Fig. 2c, f). The higher mechanical pressure necessary to maintain the structure inaccessible to $\mathrm{CH}_{4}$ molecules suggests a slightly higher internal stress induced by $\mathrm{CH}_{4}$ compared to $\mathrm{N}_{2}$. As in the case of $\mathrm{CO}_{2}$ (Fig. 2d), increases in mechanical pressure to
300 and $400 \mathrm{MPa}$ lead to a decrease in $\mathrm{CH}_{4}$ uptake indicating a gradual compression of the structure due to the mechanical pressure rather than the existence of a fully contracted pore structure.

Separation properties from single component gas adsorption. From the isotherms obtained on the three gases, the separation factors for $\mathrm{CO}_{2} / \mathrm{N}_{2}(15 / 85)$ and $\mathrm{CO}_{2} / \mathrm{CH}_{4}(50 / 50)$ mixtures at 1 bar, $303 \mathrm{~K}$, and under increasing mechanical constraint have been calculated. The separation factor is defined as the ratio of the amount adsorbed of one gas with respect to a second at their given partial pressures ${ }^{31}$, providing a valuable estimation of the separation properties of an adsorbent for a given mixture. For both separation considered, we can see that the application of mechanical pressure on the MIL-53(Al) leads to an increase of the separation factor (from 2.2 to 3 for $\mathrm{CO}_{2} / \mathrm{N}_{2}(15 / 85)$ under 0 and $100 \mathrm{MPa}$, respectively, and from 6.8 to 7.2 and 21.1 for $\mathrm{CO}_{2} / \mathrm{CH}_{4}$ (50/50) under 0,100 , and $200 \mathrm{MPa}$, respectively). More significantly, the corresponding separation factor values reach infinity above 200 and $300 \mathrm{MPa}$ for $\mathrm{CO}_{2} / \mathrm{N}_{2}$ and $\mathrm{CO}_{2} / \mathrm{CH}_{4}$, respectively, since in these conditions the gas uptake is negligible for both $\mathrm{N}_{2}$ and $\mathrm{CH}_{4}$.

An interesting approach to induce molecular sieving at subatmospheric pressure using the MIL-53(Al) has previously been proposed by Mishra et al. ${ }^{32}$. In this work, the authors carried out $\mathrm{CO}_{2}$ adsorption, and on desorption the authors did not fully reactivate the sample to the LP form. Thus, with the sample in its NP form, single-component gas adsorption isotherms evidenced negligible uptake of several gases $\left(\mathrm{N}_{2}, \mathrm{CH}_{4}, \mathrm{O}_{2}\right.$, and $\left.\mathrm{CO}\right)$. However, the range of pressure where molecular sieving can be considered is limited (around 0.6 bar for $\mathrm{N}_{2}$ and $\mathrm{CH}_{4}$ ) compared to the mechanical approach proposed here (until $10 \mathrm{bar}$ at $200 \mathrm{MPa}$ for $\mathrm{N}_{2}$ and until 5 bar at $400 \mathrm{MPa}$ for $\mathrm{CH}_{4}$ ). Also, as opposed to the work of Mishra et al. that used the non-reversible $\mathrm{CO}_{2}$ desorption behavior in the particular case of MIL-53(Al), our approach could be applied to any type of flexible adsorbents able to withstand enough mechanical pressure in order to tune their separation properties in the required gas pressure range.

The experimental results presented so far highlight the possibility to finely tune the pore size aperture of the MIL-53(Al) depending on the external mechanical pressure applied. This allowed us to control the accessibility of a given molecule to its 

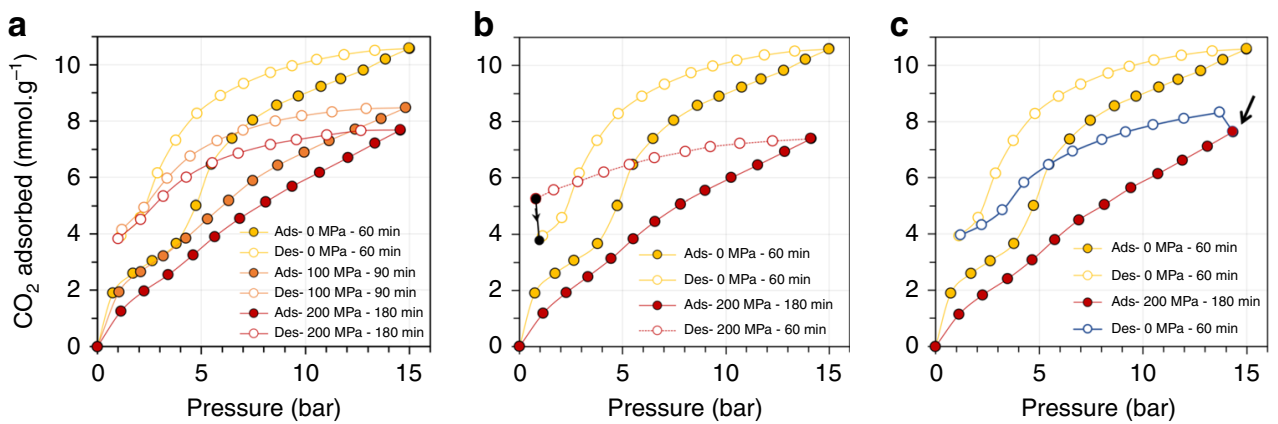

Fig. 4 Regeneration of MIL-53(Al). a-c $\mathrm{CO}_{2}$ adsorption-desorption isotherms at $303 \mathrm{~K}$ a for the unconstrained MIL-53(AI) (yellow), and under a mechanical constraint of 100 (orange) and $200 \mathrm{MPa}$ (red) with an equilibrium time set at 60,90 , and 180 minutes per point, respectively $\mathbf{b}$ under 200 MPa with an equilibrium time set at 180 minutes per point for adsorption (red full line) and 60 minutes per point for desorption (dashed red line). Filled black circles indicate the evolution of $\mathrm{CO}_{2}$ uptake when allowing the system to further equilibrate for 10 hours $\mathbf{c}$ under $200 \mathrm{MPa}$ with an equilibrium set at 180 minutes per point for adsorption (red full line) and after release of the mechanical constraint under gas pressure with an equilibrium time for desorption set at 60 minutes per point (blue line). The black arrow indicates the point on the isotherm at which the mechanical pressure has been released. b, $\mathbf{c ~} \mathrm{CO}_{2}$ adsorption-desorption isotherm for the unconstrained MIL-53(AI) (yellow) with an equilibrium time set at 60 minutes is given for comparison. a-c Filled and open circles represent adsorption and desorption, respectively.

microporosity, potentially leading to molecular sieving separations. However, caution must be taken when using single-component gas adsorption isotherms to predict the adsorption of gas mixtures in flexible MOFs as structural changes of the adsorbent may occur at different pressures for the two adsorbates ${ }^{33}$.

Separation of $\mathrm{CO}_{2} / \mathrm{N}_{2}$ and $\mathrm{CO}_{2} / \mathrm{CH}_{4}$ binary mixtures. A hybrid osmotic Monte Carlo (HOMC) simulation strategy has therefore been deployed to mimic the experimental scenario, i.e., the mechanically constrained structure is freely relaxed at all stages of adsorption. These calculations were performed on the MIL-53 framework in its Cr-version for two reasons: (i) MIL-53(Al) and MIL-53(Cr) show both similar two-step $\mathrm{CO}_{2}$ adsorption isotherms and mechanical resilience under the application of an external pressure and (ii) our previously derived flexible force field for the MIL-53 framework was fully validated for the Crversion $^{34}$. Indeed, a NP to LP phase transition of MIL-53(Cr) was predicted upon $\mathrm{CO}_{2}$ adsorption at about 5 bar in excellent agreement with the corresponding experimental data ${ }^{22}$ or under the application of an external mechanical pressure of at least $55 \mathrm{MPa}^{34}$. This magnitude of mechanical constraint was thus applied isostatically to the MIL-53(Cr) structure during the adsorption of a $\mathrm{CO}_{2} / \mathrm{N}_{2}$ mixture (molar composition 15/85) and a $\mathrm{CO}_{2} / \mathrm{CH}_{4}$ mixture (molar composition $50 / 50$ ) at $298 \mathrm{~K}$.

Figure 3 shows that for both mixtures, the amount of $\mathrm{N}_{2}$ and $\mathrm{CH}_{4}$ adsorbed by the mechanically constrained MIL-53(Cr) is negligible, while the amount of $\mathrm{CO}_{2}$ adsorbed is similar to the uptake corresponding to the filling of the NP phase. This result emphasizes an almost infinite $\mathrm{CO}_{2} / \mathrm{N}_{2}$ and $\mathrm{CO}_{2} / \mathrm{CH}_{4}$ selectivity when the sample is mechanically constrained, and this remains true even at high pressure up to 25 bar. In both cases, these simulations evidenced that the structure is maintained in a NP phase over the whole range of pressure with a unit cell volume of $1084 \AA^{3}$ associated with a pore size of $3.5 \AA$, equivalent to the dimensions of the $\mathrm{CO}_{2}$-loaded NP phase $\left(1072 \AA^{3}\right.$ ) previously reported experimentally ${ }^{36}$ and theoretically 34,35 . This confirms that the separation is driven by a size exclusion effect that hampers the adsorption of $\mathrm{N}_{2}$ and $\mathrm{CH}_{4}$ of larger kinetic diameters into the NP phase. This whole set of predictions reveals that the mechanically constrained MIL-53 is an excellent candidate for the separation of these two strategic mixtures. An adsorption cell for gas-mixture breakthrough experiments is currently under development to experimentally validate these findings.
Regeneration of the adsorbent. The other major point of our approach concerns the possibility to regenerate the material after releasing the mechanical constraint, and the desorption branches of the isotherms are key to follow for that purpose. To study the regeneration capability of the MIL-53(Al), $\mathrm{CO}_{2}$ adsorption-desorption isotherms for the unconstrained material and under various mechanical constraints were thus measured (Fig. 4a).

The isotherms obtained on the MIL-53(Al) under various mechanical constraints all display hysteresis loops indicating the presence of energy barriers for desorption as previously described $^{22}$. Comparing the results obtained with and without mechanical constraint, we observe that the amount of $\mathrm{CO}_{2}$ remaining in the pores after desorption to 1 bar is comparable (Fig. 4a). This indicates that $\mathrm{CO}_{2}$ can be desorbed from the material, whether under mechanical constraint or not. However, when increasing the mechanical pressure, the equilibrium time for adsorption/desorption becomes increasingly longer. Although it is beyond the scope of the current paper, this indicates that kinetic separations could also be envisaged with this technique as the mechanical pressure allows for a control of the mass transfer. With this in mind, the effect of releasing the mechanical pressure on the desorption diffusion rates was investigated.

Figure $4 \mathrm{~b}$ shows the $\mathrm{CO}_{2}$ adsorption-desorption isotherm recorded on the MIL-53(Al) for the unconstrained material in which a desorption time of 1 hour per point was large enough to ensure equilibrium. Adsorption isotherms are also shown for the material under constant mechanical constraint of $200 \mathrm{MPa}$, where 3 hours are required to ensure full equilibrium. However, if the desorption time is set at 1 hour for the mechanically constrained material, it can be seen that the pore emptying is not complete at the final desorption point, confirming that equilibrium is not reached (thus the dashed line). Indeed, after this final point, around 10 hours were required for the sample to finally reach equilibrium, as represented by the black filled circles. Whilst 3 hours are required to ensure full equilibrium for the mechanically constrained MIL-53(Al) under $200 \mathrm{MPa}, 80 \%$ of the gas is adsorbed in less than 30 minutes and $70 \%$ in less than 15 minutes (Supplementary Fig. 4), an important point looking at the application of such material in swing adsorption processes.

In a second experiment, the adsorption was again carried out under a mechanical constraint of $200 \mathrm{MPa}$, but the constraint was released at the end of the adsorption step (Fig. 4c). At this point, a slight increase in uptake is observed, which can be attributed to a 
relaxation of the structure to accommodate some more $\mathrm{CO}_{2}$. From this point onwards, we used a desorption time of 1 hour per point similar to the experiment with the unconstrained material, and it can be observed that the amount adsorbed after desorption exactly matches that for the unconstrained sample. This is a key result indicating that the regeneration properties of the adsorbent are retrieved when the mechanical pressure is released.

\section{Discussion}

This fundamental exploration of the adsorption behavior of the flexible MIL-53 under mechanical constraint demonstrates the possibility to finely tune its pore size aperture during both the adsorption and desorption processes. The fine control of the pore size with the magnitude of applied mechanical pressure allows (i) the separation properties to be optimized for a given mixture, while (ii) improving the regeneration capability and the desorption diffusion rates of flexibles MOFs compared to rigid ultramicroporous materials. Such methodology is highly attractive for the molecular sieving separations of the most challenging gas mixtures, but further works have to be conducted to study its large-scale application. The key challenge to address is to ensure a uniform distribution of the mechanical pressure on the powder bed at a large scale, in order to spatially achieve similar separation properties in the adsorption column. Nonetheless, the proof-ofconcept presented here highlights the possibility to precisely and reversibly reconfigure the microporous network of flexible MOFs in response to an external stimulus. This approach paves the way for the development of new separation processes, but could also be relevant for any applications where the in situ control of an absorbent porosity is of interest.

\section{Methods}

Adsorption measurements under applied mechanical pressure. An experimental setup for the in situ measurement of gas adsorption isotherms under controlled mechanical constraint was specially designed and used (Supplementary Fig. 1). First, a sample-cell has been developed in order to maintain the gas pressure when applying an external mechanical constraint (Supplementary Fig. 1-Photo). The cell is based on a die press used to make pellets for infrared spectroscopy with the particularity to use O-rings to maintain the system leak-tight to gas pressure. An inlet and valve system to allow vacuum and gas input has equally been built onto the cell. The leak tests have shown a perfect sealing until 20 bar. A CrushIR digital hydraulic press from PIKE technologies is used to load the mechanical pressure up to 15 ton. This is equivalent to around $3000 \mathrm{MPa}$ on the sample as the contact surface of the piston is of $0.5 \mathrm{~cm}^{2}\left(1\right.$ ton $\left.\mathrm{cm}^{2}=98.1 \mathrm{MPa}\right)$.

Prior to the introduction in the cell, the MIL-53(Al) sample prepared following the synthesis route previously reported ${ }^{21}$ is activated ex situ at $473 \mathrm{~K}$ for $16 \mathrm{~h}$ under a dynamic vacuum of $10^{-3} \mathrm{mbar}$. It is then transferred to the sample-cell in a glovebox to avoid any contamination from the ambient atmosphere. The cell is placed under the hydraulic press and then connected to an adsorption manometry system for the measurement of adsorption isotherms. The entire system is located in a thermally controlled cage, and the temperature maintained at $303 \mathrm{~K}$. The gas dosing system was designed and constructed in-house using Swagelok VCR 1/4" stainless steel components with a series of pneumatic valves and consists of a gas inlet line, a measurement manifold and a gas evacuation system. The leak-tightness is ensured by copper ring seals, and the system is designed to withstand pressures up to $200 \mathrm{bar}$, although the pressure gauges currently installed (Mensor CPT6000 series) have a maximum pressure of 40 bar. The gas injection system is completely automated via homemade software and interface.

The measurement manifold consists of the sample cell and a reference volume with both a low-pressure gauge (to 1 bar) and a high-pressure gauge (from 1 to 40 bar) for optimal accuracy on the entire pressure range. The reference volume was first determined by the expansion of He from a calibrated volume of $26,069 \mathrm{~cm}^{3}$. The adsorption measurements are carried out based on the standard manometric adsorption technique. A gas dose is introduced into the reference volume through valve V2 and allowed to stabilize before being brought into contact with the sample by opening valve V3 (Supplementary Fig. 1). The amount of gas introduced into the reference volume and remaining in the system after adsorption is calculated via REFPROP software, and the amount adsorbed is determined from the mass balance. In order to calculate the amount adsorbed, it is equally necessary to know the precise dead volume of the cell containing the sample. This is obtained by an experiment involving an inert gas, which is assumed not to adsorb (Helium) prior to each adsorption experiment.
X-ray diffraction. XRD patterns were acquired using a powder INEL diffractometer at CINAM laboratory (UMR 7325 CNRS AMU). The incident beam was monochromatic with CuKal radiation $(\lambda=1.5406 \AA)$. The samples were introduced into thin-walled glass capillaries (inner diameter $0.4 \mathrm{~mm}$ ). The sample impregnation in ethanol was done in order to fully open the structure (LP phase) and avoid any LP/NP phase coexistence. It was done directly by dropping a few droplets of ultrapure ethanol inside the capillary. Data collection was done using a curved position sensitive detector over a total angular range $2 \theta$ between $0.29^{\circ}$ and $107.42^{\circ}$ by steps of $0.029^{\circ}$. During the acquisition, the capillary was mounted on a rotating holder to avoid any preferential orientation. The acquisition time was set to $1800 \mathrm{~s}$.

Modeling. The simulation box consisted of 32 unit cells of the conventional LP structure of MIL-53(Cr) built from the crystallographic coordinates previously reported by X-ray powder diffraction (XRPD) study ${ }^{21}$. The corresponding box lengths are $L_{x}=33.2 \AA, L_{y}=52.8 \AA$, and $L_{z}=26.8 \AA$. The MIL-53(Cr) framework was described by our previously derived flexible forcefield, including intramolecular and inter-molecular potential terms ${ }^{34} . \mathrm{CH}_{4}, \mathrm{CO}_{2}, \mathrm{~N}_{2}$ were treated with the standard microscopic models reported in the literature ${ }^{36-38}$. The MIL-53(Cr)/ gas molecules interactions were treated by a Lennard-Jones (LJ- and Coulombic terms published elsewhere ${ }^{34}$ with the cross-term LJ parameters obtained using the mixing Lorentz-Berthelot rules). Ewald summation was used for calculating the electrostatic interactions, and the short-range interactions were truncated at $12 \AA$. The HOMC corresponds to a GCMC scheme combined with a MD move in $N \sigma T$ ensemble (where $N$ is the number of molecules, $T$ the temperature and $\sigma$ the isotropic constraint) to allow the anisotropic changes of both shape and volume of the MOF framework ${ }^{39}$. The Nose-Hoover thermostat and barostat were used with a relaxation time of 0.5 ps. MD moves were conducted for 100 ps with a time step of $2 \mathrm{fs}$. The equations of motion were integrated using the velocity Verlet scheme. The MC procedure in the grand canonical ensemble corresponds to 5000 steps such that the ratios for each trial moves were defined as follows: 0.2004 for the translation, 0.2004 for the rotation, 0.599 for the insertion/deletion and 0.0002 for the MD move. A HOMC run corresponds to 100 steps corresponding to a sampled MD time of $1.0 \mathrm{~ns}$ and 500,000 MC steps. To validate our findings, several HOMC simulations with different MD and MC steps pairs have been tested in order to confirm that the simulations do not depend on the MC or MD steps number. In addition, we have conducted a very long time simulation $\left(1 \times 10^{5}\right.$ HOMC steps i.e., $\sim 100 \mathrm{~ns}$ and $500 \times 10^{6} \mathrm{MC}$ steps) to confirm the stability of the HOMC algorithm.

\section{Data availability}

The data that support the findings of this study are available on request from the corresponding author.

Received: 13 August 2019; Accepted: 8 February 2020; Published online: 05 March 2020

\section{References}

1. Sholl, D. S. \& Lively, R. P. Seven chemical separations to change the world. Nat. News 532, 435 (2016).

2. U.S. Department of Energy's Office of Energy Efficiency and Renewable Energy, Industrial Technologies Program. Materials Research for Separations Technologies: Energy and Emissions Reduction (US Department of Energy, 2005).

3. Adil, K. et al. Gas/vapour separation using ultra-microporous metal-organic frameworks: insights into the structure/separation relationship. Chem. Soc. Rev. 46, 3402-3430 (2017).

4. Bhatt, P. M. et al. Fine-tuned fluorinated MOF addresses the needs for trace $\mathrm{CO}_{2}$ removal and air capture using physisorption. J. Am. Chem. Soc. 138, 9301-9307 (2016)

5. Cadiau, A., Adil, K., Bhatt, P. M., Belmabkhout, Y. \& Eddaoudi, M. A metalorganic framework-based splitter for separating propylene from propane. Science 353, 137-140 (2016)

6. Assen, A. H. et al. Ultra-tuning of the rare-earth fcu-MOF aperture size for selective molecular exclusion of branched paraffins. Angew. Chem. Int. Ed. 54, 14353-14358 (2015).

7. Schneemann, A. et al. Flexible metal-organic frameworks. Chem. Soc. Rev. 43, 6062-6096 (2014).

8. Férey, G. \& Serre, C. Large breathing effects in three-dimensional porous hybrid matter: facts, analyses, rules and consequences. Chem. Soc. Rev. $\mathbf{3 8}$ 1380-1399 (2009).

9. Krause, S. et al. A pressure-amplifying framework material with negative gas adsorption transitions. Nature 532, 348-352 (2016).

10. Coudert, F. X. Responsive metal-organic frameworks and framework materials: under pressure, taking the heat, in the spotlight, with friends. Chem. Mater. 27, 1905-1916 (2015). 
11. Matsuda, R. Selectivity from flexibility. Nature 509, 434-435 (2014).

12. Liu, Y. et al. Reversible structural transition in MIL-53 with large temperature hysteresis. J. Am. Chem. Soc. 130, 11813-11818 (2008).

13. Modrow, A., Zargarani, D., Herges, R. \& Stock, N. The first porous MOF with photoswitchable linker molecules. Dalton Trans. 40, 4217-4222 (2011).

14. Ghoufi, A., Benhamed, K., Boukli-Hacene, L. \& Maurin, G. Electrically induced breathing of the MIL-53(Cr) metal-organic framework. ACS Cent. Sci. 3, 394-398 (2017).

15. Beurroies, I. et al. Using pressure to provoke the structural transition of metalorganic frameworks. Angew. Chem. Int. Ed. 49, 7526-7529 (2010).

16. Rodriguez, J., Beurroies, I., Loiseau, T., Denoyel, R. \& Llewellyn, P. L. The direct heat measurement of mechanical energy storage metal-organic frameworks. Angew. Chem. Int. Ed. 54, 4626-4630 (2015).

17. Yot, P. G. et al. Impact of the metal centre and functionalization on the mechanical behaviour of MIL-53 metal-organic frameworks. Eur. J. Inorg. Chem. 27, 4424-4429 (2016).

18. Yot, P. G. et al. Metal-organic frameworks as potential shock absorbers: the case of the highly flexible MIL-53(Al). Chem. Comm. 50, 9462-9464 (2014).

19. Yot, P. G. et al. Mechanical energy storage performance of an aluminium fumarate metal-organic framework. Chem. Sci. 7, 446-450 (2016).

20. Mason, J. A. et al. Methane storage in flexible metal-organic frameworks with intrinsic thermal management. Nature 527, 357-361 (2015).

21. Serre, C. et al. Very large breathing effect in the first nanoporous chromium (III)-based solids: MIL-53 or Cr-III $(\mathrm{OH}) \bullet\left\{\mathrm{O}_{2} \mathrm{C}-\mathrm{C}_{6} \mathrm{H}_{4}-\mathrm{CO}_{2}\right\} \bullet\left\{\mathrm{HO}_{2} \mathrm{C}-\mathrm{C}_{6} \mathrm{H}_{4}\right.$ $\left.\mathrm{CO}_{2} \mathrm{H}\right\}(\mathrm{x}) \cdot \mathrm{H}_{2}$ Oy. J. Am. Chem. Soc. 124, 13519-13526 (2002).

22. Bourrelly, S. et al. Different adsorption behaviors of methane and carbon dioxide in the isotypic nanoporous metal terephthalates MIL-53 and MIL-47. J. Am. Chem. Soc. 127, 13519-13521 (2005).

23. McClellan, A. L. \& Harnsberger, H. F. Cross-sectional areas of molecules adsorbed on solid surfaces. J. Colloid Interface Sci. 23, 577-599 (1967).

24. Loiseau, T. et al. A rationale for the large breathing of the porous aluminum terephthalate (MIL-53) upon hydration. Chem. Eur. J. 10, 1373-1382 (2004).

25. Dundar, E. et al. Modeling of adsorption of $\mathrm{CO}_{2}$ in the deformed pores of MIL-53 (Al). J. Mod. Model 23, 101-110 (2017).

26. Ramsahye, N. et al. Probing the adsorption sites for $\mathrm{CO}_{2}$ in MIL-53(Al, Cr) and MIL-47(V) Metal Organic Framework materials by density functional theory. J. Phys. Chem. C 112, 514-520 (2008).

27. Ramsahye, N. et al. $\mathrm{CO}_{2}$ adsorption in the hybrid porous MIL-53(Al) material, a grand canonical Monte Carlo simulation compared to microcalorimetry. Phys. Chem. Chem. Phys. 9, 1059 (2007).

28. Ramsahye, N. et al. On the breathing effect of the aluminium terephtalate MIL-53 upon $\mathrm{CO}_{2}$ adsorption, a computational study compared to experiments. Chem. Commun. 31, 3261 (2007).

29. Majmudar, T. S. \& Behringer, R. P. Contact force measurements and stressinduced anisotropy in granular materials. Nature 435, 1079-1082 (2005).

30. Farhadi, S., Zhu, A. Z. \& Behringer, R. P. Stress relaxation for granular materials near jamming under cyclic compression. Phys. Rev. Lett. 115, 188001 (2015).

31. Rouquerol, J., Rouquerol, F., Llewellyn, P., Maurin, G. \& Sing, K. S. W. Adsorption by Powders and Porous Solids: Principles, Methodology and Applications, 2nd edn (Elsevier, Amsterdam, 2014).

32. Mishra, P., Edubilli, S., Uppara, H. P., Mandal, B. \& Gumma, S. Effect of adsorbent history on adsorption characteristics of MIL-53 (Al) metal organic framework. Langmuir 29, 12162-12167 (2013).

33. Fraux, G., Boutin, A., Fuchs, A. H. \& Coudert, F. X. On the use of the IAST method for gas separation studies in porous materials with gate-opening behavior. Adsorption 24, 233-241 (2018).

34. Ghouf, A. et al. Comparative guest, thermal, and mechanical breathing of the porous metal organic framework MIL-53(Cr): a computational exploration supported by experiments. J. Phys. Chem. C 116, 13289-13295 (2012).

35. Salles, F. et al. Molecular dynamics simulations of breathing MOFs: structural transformations of MIL-53(Cr) upon thermal activation and $\mathrm{CO}_{2}$ adsorption. Angew. Chem. Int. Ed. 47, 8487-8491 (2008).
36. Serre, C. et al. An explanation for the very large breathing effect of a metalorganic framework during $\mathrm{CO}_{2}$ adsorption. Adv. Mater. 19, 2246-2251 (2007)

37. Rosenbach, N. et al. Quasi-elastic neutron scattering and molecular dynamics study of methane diffusion in metal organic frameworks MIL-47(V) and MIL 53(Cr). Angew. Chem. Int. Ed. 47, 6611-6615 (2008).

38. Harris, J. G. \& Yung, K. H. J. Carbon dioxides's liquid-vapor coexistence curve and critical properties as predicted by a simple molecular model. J. Phys. Chem. 99, 12021-12024 (1995).

39. Ghoufi, A. \& Maurin, G. Hybrid monte carlo simulations combined with a phase mixture model to predict the structural transitions of a porous metalorganic framework material upon adsorption of guest molecules. J. Phys. Chem. C 114, 6496-6502 (2010).

\section{Acknowledgements}

This research was supported by the French Research Agency (ANR MEACOPA, no. ANR-17-CE29-0003-02). N.C. would like to thank Total S. A. for support. In addition, we thank C. Serre for providing the sample, Y. Cecere for his participation in the design and conception of the adsorption cell and V. Heresanu for providing access to the X-ray diffractometer and his help in performing the experiments. We also thank R. Denoyel, P. Valiorgue, R. Pellenq, and T. Divoux for discussions and comments.

\section{Author contributions}

P.L.L. had the initial idea. N.C., P.L.L., S.B., B.K., and G.M. formulated the project. N.C. designed and developed the adsorption cell and the experimental adsorption setup performed adsorption experiments, and analyzed adsorption data. N.C. performed calculations of the separation factors. M.-V.C. collected and analyzed X-ray diffraction data A.G. and G.M. carried out the molecular simulations. N.C., G.M., and P.L.L. wrote the paper, and all authors contributed to revising the paper.

\section{Competing interests}

The authors declare no competing interests.

\section{Additional information}

Supplementary information is available for this paper at https://doi.org/10.1038/s41467020-15036-y.

Correspondence and requests for materials should be addressed to N.C.

Peer review information Nature Communications thanks Sasidhar Gumma, Brian Space and other, anonymous, reviewers for their contributions to the peer review of this work

Reprints and permission information is available at http://www.nature.com/reprints

Publisher's note Springer Nature remains neutral with regard to jurisdictional claims in published maps and institutional affiliations.

Open Access This article is licensed under a Creative Commons Attribution 4.0 International License, which permits use, sharing, adaptation, distribution and reproduction in any medium or format, as long as you give appropriate credit to the original author(s) and the source, provide a link to the Creative Commons license, and indicate if changes were made. The images or other third party material in this article are included in the article's Creative Commons license, unles indicated otherwise in a credit line to the material. If material is not included in the article's Creative Commons license and your intended use is not permitted by statutory regulation or exceeds the permitted use, you will need to obtain permission directly from the copyright holder. To view a copy of this license, visit http://creativecommons.org/ licenses/by/4.0/.

(C) The Author(s) 2020, corrected publication 2021 